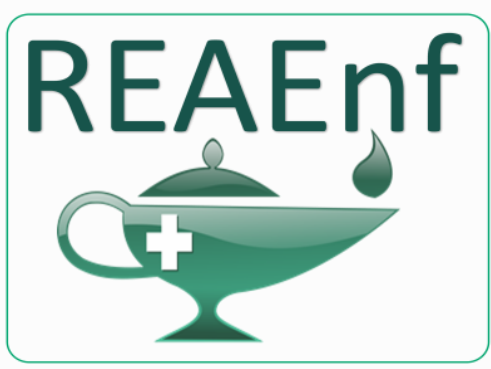

Revista Eletrônica Acervo Enfermagem
ARTIGO ORIGINAL

Recebido em: 4/2020

Aceito em: $5 / 2020$

Publicado em: 6/2020

\title{
Infecção de trato urinário associada ao cateterismo vesical de demora na população idosa: classificações de enfermagem
}

\author{
Urinary tract infection associated with delayed bladder catheterization in the elderly \\ population: nursing classifications
}

\section{Infección de tracto urinario asociada al cateterismo vesical de demora en la población anciana: clasificaciones de enfermería}

Myria Ribeiro da Silva ${ }^{1 *}$, Talita Hevilyn Ramos da Cruz Almeida ${ }^{1}$, Talita Rosa dos Santos ${ }^{1}$, Emilaine Santos Souza ${ }^{1}$, Ricardo Matos Santana ${ }^{1}$.

\begin{abstract}
Resumo: Esse artigo buscou identificar os diagnósticos de enfermagem mais frequentes utilizando o NANDA - I, sugerir resultados e intervenções de enfermagem, além de propor protocolos sobre prevenção de Infecções do Trato Urinário (ITU). Trata-se de estudo descritivo, qualiquantitativo, o qual a coleta de dados ocorreu nos anos de 2014 e 2015, mediante fontes secundárias encontradas em um hospital da Região Sul da Bahia - Brasil. Foram identificados 155 pacientes idosos internados, sendo que 87 deles foram diagnosticados com ITU associado ao Cateterismo Vesical de Demora (CVD). Entre os diagnósticos de enfermagem identificados destacaram-se: Proteção ineficaz; Risco de Infecção e Risco de lesão em $100 \%$ dos pacientes; Hipertermia e Dor Aguda em 90\% dos casos. Com base nos diagnósticos identificados foram planejadas as intervenções, dentre os quais estão: Controle de Infecção, Tratamento da Febre e o Controle da Dor. O conhecimento sobre as classificações de Enfermagem na população idosa, com ITU associado ao uso do CVD é imprescindível para subsidiar a assistência, bem como fundamentar as ações em bases teóricas científicas seguras, além de contribuir de forma efetiva para a valorização do conhecimento científico, sustentabilidade do aporte teórico-prático e autonomia da profissão na prática assistencial.
\end{abstract}

Palavras-chave: Idoso, Classificações de enfermagem, Cateter vesical de demora.

\begin{abstract}
This article sought to identify the most frequent nursing diagnoses using NANDA - I, to suggest results and nursing interventions, in addition to proposing protocols on prevention of Urinary Tract Infection (UTI). This is a descriptive, qualitative and quantitative study, which data collection took place in 2014 and 2015, using secondary sources found in a hospital in the Southern Region of Bahia - Brazil. 155 inpatient elderly patients identified, 87 of whom were diagnosed with UTI associated with Indwelling Bladder Catheterization (IBC). Among the nursing diagnoses identified, the following stand out: Ineffective protection; Risk of infection and risk of injury in 100\% of patients; Hyperthermia and Acute Pain in $90 \%$ of cases. Based on the diagnoses identified, interventions were planned, among which are: Infection Control, Fever Treatment and Pain Control. Knowledge about nursing classifications in the elderly population, with UTI associated with the use of IBC is essential for subsidiary assistance, as well as being based on actions based on scientific theoretical security, in addition to making an effective contribution to the valorization of scientific knowledge, sustainability. a theoretical-practical contribution and scope of the profession in care practice.
\end{abstract}

Keywords: Elderly, Nursing ratings, Indwelling catheters.

1 Universidade Estadual de Santa Cruz (UESC), Ilhéus - Bahia. *E-mail: myriarib@uol.com.br 
Resumen: Este artículo buscaba identificar los diagnósticos de enfermería más frecuentes utilizando NANDA - I, también para sugerir resultados e intervenciones de enfermería, además de proponer protocolos sobre prevención de Infecciones del Tracto Urinario (ITU). Este es un estudio descriptivo, cualitativo y cuantitativo, cuya recopilación de datos tuvo lugar en 2014 y 2015, utilizando fuentes secundarias encontradas en un hospital en la Región Sur de Bahía - Brasil. Se identificaron 155 pacientes hospitalizados de edad avanzada, 87 de los cuales fueron diagnosticados con infección urinaria asociada con Cateterización Vesical (CV). Entre los diagnósticos de enfermería identificados, se destacan los siguientes: protección ineficaz; Riesgo de infección y riesgo de lesiones en el 100\% de los pacientes; Hipertermia y dolor agudo en el $90 \%$ de los casos. En función de los diagnósticos identificados, se planificaron intervenciones, entre las que se encuentran: Control de infecciones, Tratamiento de la fiebre y Control del dolor. El conocimiento sobre las clasificaciones de enfermería en la población de edad avanzada, con ITU asociada con el uso de ECV es esencial para apoyar la atención, así como para basar las acciones en bases teóricas científicas seguras, además de contribuir de manera efectiva a la mejora del conocimiento científico, la sostenibilidad La contribución teórico-práctica y la autonomía de la profesión en la práctica asistencial.

Palabras clave: Ancianos; Clasificaciones de enfermería; Cateter vesical de demora.

\section{INTRODUÇÃO}

A Infecção do Trato Urinário (ITU) é caracterizada pela presença de microrganismos patogênicos nas vias urinárias. $O$ trato urinário saudável é estéril acima da uretra. Para que ocorra a infecção, as bactérias precisam ter acesso à bexiga, fixar-se ao epitélio do trato urinário e colonizá-lo. Essa é uma das causas prevalentes de Infecções Relacionadas à Assistência à Saúde (IRAS) de grande potencial preventivo, visto que a maioria está relacionada ao Cateter Vesical de Demora (CVD). Aproximadamente $16-25 \%$ dos pacientes de um hospital serão submetidos a cateterismo vesical, de alívio ou de demora, em algum momento de sua hospitalização, muitas vezes sob indicação clínica equivocada ou inexistente e até mesmo sem conhecimento médico (BRASIL, 2017).

Arrais ELM, et al. (2017) apontam os seguintes fatores de risco para a ITU: Idade avançada, sexo feminino (por apresentar meato uretral de menor comprimento e mais próximo do ânus), disfunções anatômicas e comorbidades. Já os principais fatores predisponentes que afetam esse grupo são: uropatias obstrutivas da próstata em homens, pouco esvaziamento da bexiga por prolapso uterino nas mulheres, declínio do status cognitivo, institucionalização e cateterismos intermitentes ou de demora. Esses fatores se configuram em pior prognósticos para o idoso, além de ser a causa mais comum de sepse em indivíduos acima de 60 anos (BRASIL, 2017; ARRAIS ELM, et al., 2017).

A ITU relacionada ao uso de CVD é caracterizada como um problema de saúde pública, uma vez que, mesmo quando implementada a técnica asséptica de inserção do cateter e manutenção do dispositivo, o risco de infecção pode ser de 5 a $10 \%$ a cada dia em uso. Agrega-se a isso que a prevenção e o controle devem ser intensificados quando estes cuidados forem implementados na população vulnerável, como por exemplo, a idosa (BRASIL, 2017). Nesse contexto, é imprescindível a atuação do enfermeiro enquanto gestor e prestador direto dos cuidados, se fazendo necessário planejar a assistência, considerando as singularidades dos sujeitos, empregando-se a Sistematização da Assistência de Enfermagem (SAE) através da execução do Processo de Enfermagem, como previsto na Resolução COFEN 358/2009 (COFEN, 2009).

A Sistematização da Assistência de Enfermagem (SAE) configura-se como uma metodologia para organizar e sistematizar o cuidado, com base nos princípios do método científico. Tem como objetivos identificar as situações de saúde-doença e as necessidades de cuidados de enfermagem, bem como subsidiar as intervenções de promoção, prevenção, recuperação e reabilitação da saúde do indivíduo, família e comunidade (SCHMITZEL EL, et al., 2016).

O Processo de Enfermagem constitui-se de cinco etapas, sendo elas a Coleta de Dados, o Diagnóstico de Enfermagem, o Planejamento, a Implementação e a Avaliação. Ao enfermeiro compete liderar a aplicação deste instrumento em todas as instituições em que há atendimento de enfermagem, fiscalizando e

REAEnf/EJNC | Vol. 3 | e3540 | DOI: https://doi.org/10.25248/REAenf.e3540.2020 Página 2 de 8 
norteando os técnicos e auxiliares de enfermagem, sendo de competência privativa do enfermeiro o diagnóstico e a prescrição das intervenções (COFEN, 2009).

Nesse sentido, as classificações de enfermagem surgiram com a premissa de permitir uma linguagem padronizada e científica para as práticas de enfermagem. Atualmente, existem várias classificações, destacam-se nesse estudo as classificações internacionais de Enfermagem: Diagnóstico (NANDA), Intervenção (NIC) e Resultados (NOC). Cada uma é utilizada em determinada fase do processo de enfermagem, sendo que todas elas são interligadas, de forma a contribuir para a adequação do cuidado prestado, de acordo com a situação clínica do paciente, comunicação entre os profissionais e a documentação da prática clínica (FERREIRA AM, et al., 2016).

Tendo em vista a complexidade da temática, questionou-se: quais são os diagnósticos, resultados e intervenções de enfermagem presentes em pacientes idosos internados com ITU associada ao CVD?

O despertar para este objeto de estudo se deu pela relevância do tema proposto, bem como pela necessidade de contribuir com a produção de conhecimentos que propiciem melhoria dos cuidados prestados aos idosos com vistas à promoção da saúde integral pela sistematização desse cuidado. Deste modo, o objetivo deste estudo foi identificar os principais diagnósticos, intervenções e resultados de enfermagem descritos pela North American Nursing Diagnosis Association (NANDA), Nursing Outcomes Classification (NOC), Nursing Intervention Classification (NIC), para pacientes idosos internados.

\section{MÉTODOS}

Estudo descritivo-exploratório, com abordagem quantitativa e qualitativa, realizado nas unidades de internação, em um hospital público localizado na Região Sul do estado da Bahia, Brasil. Com base em amostragem de conveniência, adotou-se como critérios de seleção clientes idosos em uso de CVD, admitidos no referido hospital, no período de fevereiro a setembro de 2016, e que concordaram em participar da pesquisa preenchendo e assinando o Termo de Consentimento Livre e Esclarecido.

Os critérios para inclusão na pesquisa foram pessoas com idade igual ou superior a 60 anos que foram submetidas ao cateterismo vesical de demora, sendo verificado o nível de consciência mediante a aplicação da Escala de Coma Glasgow, com escore igual a 15. Para a coleta de dados foram realizados anamnese e exame clínico e como fonte secundária de dados utilizou-se os prontuários dos clientes.

O estudo foi submetido e aprovado pelo Comitê de Ética em Pesquisa da Universidade Estadual de Santa Cruz/UESC, parecer ํㅜ 436.807.

Os diagnósticos, resultados e intervenções/atividades foram confrontados com a literatura e avaliados por cinco peritos com expertise na área.

A análise foi realizada no Statistical Package for Social Science (SPSS), versão 20.0, por meio de frequências absolutas e relativas, de modo a se obter a frequência dos diagnósticos, resultados e intervenções de enfermagem com o objetivo de se conseguir um perfil do Processo de Enfermagem. Consideraram-se principais os títulos dos diagnósticos, resultados e intervenções/atividades formulados para mais de $50 \%$ dos participantes do estudo. Os títulos de diagnósticos de enfermagem foram apresentados segundo os domínios da NANDA-I, de modo a favorecer a identificação das áreas de atenção à população idosa.

\section{RESULTADOS}

Foram identificados 155 pacientes idosos internados, com média de idade de 70 anos e com tempo médio de internação de 18 dias. Destes pacientes institucionalizados, 87 foram diagnosticados com ITU associado ao cateter vesical de demora (CVD).

Foi observado como aponta a Tabela 1, que a maioria dos pacientes com ITU associada ao Cateter Vesical de Demora é do sexo feminino com $56,32 \%$ dos casos e o sexo masculino com $43,68 \%$ dos casos,

REAEnf/EJNC | Vol. 3 | e3540 | DOI: https://doi.org/10.25248/REAenf.e3540.2020 Página 3 de 8 
em uma somatória de 87 casos analisados que corresponderam a 56,12\% do total de 155 idosos internados. 70\% das ITU's analisadas (corresponde a 61 idosos) foram causadas pelo agente etiológico Escherichia coli, os demais foram provocados pelo Proteus sp.

Tabela 1 - Caracterização de idosos com ITU, segundo gênero e mês. Bahia, 2016.

\begin{tabular}{cccc}
\hline Mês & $\mathbf{F ~ ( \% )}$ & $\mathbf{M}(\%)$ & $\mathbf{N}(\%)$ \\
\hline Fev & $7(14,28)$ & $6(15,79)$ & $13(14,94)$ \\
Mar & $6(12,24)$ & $4(10,52)$ & $10(11,49)$ \\
Abr & $5(10,20)$ & $4(10,52)$ & $9(10,34)$ \\
Mai & $7(14,28)$ & $6(15,79)$ & $13(14,94)$ \\
Jun & $6(12,24)$ & $4(10,52)$ & $10(11,49)$ \\
Jul & $7(14,28)$ & $3(07,90)$ & $10(11,49)$ \\
Ago & $7(14,28)$ & $5(13,16)$ & $12(13,79)$ \\
Set & $4(08,16)$ & $6(15,79)$ & $10(11,49)$ \\
\hline Total & $\mathbf{4 9 ( 5 6 , 3 2 )}$ & $\mathbf{3 8 ( 4 3 , 6 8 )}$ & $\mathbf{8 7}(\mathbf{5 6 , 1 2 )}$ \\
\hline
\end{tabular}

Fonte: Silva MR, et al., 2020.

Quanto as causas de base do internamento hospitalar (de acordo com a Classificação Internacional de Doenças e Problemas Relacionados à Saúde - CID 10) destacam-se as doenças de cunho neurológico com 23,88\%; Urológico 20,65\%; Ortopédico 17,42\%; Cardiológico 14,19\%; Endócrino 11,61\%, Gastroentérico $7,74 \%$, outros fatores corresponderam a $4,51 \%$.

De acordo com a investigação de enfermagem foram identificados os diagnósticos de enfermagem mais relevantes segundo as classificações NANDA, NIC, NOC que corresponderam: Proteção Ineficaz; Risco de Infecção; Risco de Lesão em 100\% dos pacientes; Hipertermia 90\% dos casos e Dor aguda em $80 \%$. Para melhor compreensão foram descritos os domínios, fatores relacionados e características definidoras segundo a taxonomia II da NANDA- I (Quadro 1).

Quadro 1 - Diagnósticos de Enfermagem, Fatores relacionados, Características definidoras e Domínios frequente nos pacientes internados com ITU/CVD. Bahia, 2016.

\section{Domínio 1: Promoção à saúde}

- PROTEÇÃO INEFICAZ

Características Definidoras: Deficiência na Imunidade; Presença da Infecção do Trato urinário.

Fatores Relacionados: Extremos de idade.

Domínio 11: Segurança/Proteção

- RISCO DE INFECÇÃO

Fatores Relacionados: Instrumentação do trato urinário; Procedimento invasivo (inserção do cateter vesical demora); Existência de infecção no trato urinário baixo com possível ascensão para o Trato Urinário Superior (pielonefrite)

- RISCO DE LESÃO

Fatores Relacionados: Trauma tissular; Pressão, abrasão e lesão da mucosa durante a passagem da sonda vesical.

- HIPERTERMIA

Características Definidoras: Aumento na temperatura corporal acima dos parâmetros normais; Pele quente ao toque.

Fatores Relacionados: Processo infeccioso; Inflamação.

\section{Domínio 12: Conforto}

- DOR AGUDA

Características Definidoras: Relato verbal de dor em região supra púbica.

Fatores Relacionados: Inflamação e infecção da uretra, bexiga e outras estruturas do trato urinário.

Fonte: Silva MR, et al., 2020. Baseado em Herdman TH, Kamitsuru S, 2017.

No Quadro 2 podem ser observados que, para cada diagnóstico de enfermagem, foram relacionados os resultados com indicadores e intervenções. Dentre as principais intervenções de enfermagem têm-se: 
remoção do cateter assim que possível, a higienização das mãos antes e após manipulação do cateter vesical de demora, manter técnica asséptica e monitorar a temperatura do paciente frequentemente.

Quadro 2 - Diagnósticos, resultados e intervenções de enfermagem (NANDA, NOC e NIC). Bahia, 2016.

\begin{tabular}{|c|c|c|}
\hline Diagnósticos & Resultados & Indicadores \\
\hline Proteção Ineficaz & $\begin{array}{l}\text { Conhecimento: controle de } \\
\text { infecção }\end{array}$ & $\begin{array}{l}\text { Importância higienização das mãos; } \\
\text { Fatores que contribuem para transmissão. }\end{array}$ \\
\hline \multicolumn{3}{|c|}{$\begin{array}{l}\text { Intervenções NIC } \\
\text { Controle de Infecção: } \\
\text { - Usar cateterização intermitente para reduzir risco de ITU; } \\
\text { - } \quad \text { Administrar antibiótica, conforme apropriado. } \\
\text { Cuidados com Sondas/drenos: } \\
\text { - } \quad \text { Manter o sistema de drenagem urinária fechado; } \\
\text { - Hemover o cateter, assim que possível; } \\
\end{array}$} \\
\hline Risco de Infecção & $\begin{array}{l}\text { Controle de Risco: processo } \\
\text { infeccioso. }\end{array}$ & $\begin{array}{l}\text { Identificação de risco de infecção em } \\
\text { situações cotidianas. }\end{array}$ \\
\hline \multicolumn{3}{|c|}{$\begin{array}{l}\text { Intervenções NIC } \\
\text { Proteção contra Infecção: } \\
\text { - Examinar a pele e mucosas em busca de hiperemia; } \\
\text { - } \quad \text { Monitorar a vulnerabilidades a infecção; } \\
\text { - Orientar ao paciente e a família maneiras de evitar infecção. }\end{array}$} \\
\hline Risco de Lesão & $\begin{array}{l}\text { Integridade Tissular: pele e } \\
\text { mucosa ou Detecção de riscos }\end{array}$ & $\begin{array}{l}\text { Lesão na Mucosa; } \\
\text { Lesão da pele. }\end{array}$ \\
\hline \multicolumn{3}{|c|}{$\begin{array}{l}\text { Intervenções NIC } \\
\text { Sondagem Vesical: } \\
\text { - Manter técnica asséptica; } \\
\text { - Usar sonda de menor tamanho, conforme apropriado; } \\
\text { - } \quad \text { Fixar a sonda corretamente evitando traumas; } \\
\text { - } \text { Atentar para o gel lubrificante estéril e de uso único. }\end{array}$} \\
\hline Hipertermia & Sinais Vitais & Temperatura corporal \\
\hline \multicolumn{3}{|c|}{$\begin{array}{l}\text { Intervenção da NIC } \\
\text { Tratamento da Febre: } \\
\text { - } \quad \text { Monitorar a temperatura com a frequência adequada; } \\
\text { - Dar medicação adequada para prevenir ou controlar calafrios. }\end{array}$} \\
\hline Dor Aguda & Nível de Dor & Dor relatada; expressão facial de dor \\
\hline \multicolumn{3}{|c|}{$\begin{array}{l}\text { Intervenções da NIC } \\
\text { Controle da Dor } \\
\text { - Realizar uma avaliação completa da dor, incluindo local, característica, início/ duração, } \\
\text { frequência, qualidade, intensidade e gravidade, além de fatores precipitados. } \\
\text { - Assegurar que o paciente receba cuidados precisos de analgesia }\end{array}$} \\
\hline
\end{tabular}

Fonte: Silva MR, et al., 2020. Baseado em Herdman TH, Kamitsuru S, 2017; Bulechek GM, et al., 2016; Moorhead S, et al., 2016.

\section{DISCUSSÃO}

A ITU associada ao CVD foi mais prevalente em mulheres idosas, com 49 casos. Nos homens, foram identificados 38 casos, mostrando que não há uma diferença expressiva no número de infecções relacionadas ao sexo, no entanto, as infecções urinárias, neste caso, possivelmente estão mais relacionadas ao processo de envelhecimento e ao CVD do que com a própria alteração da anatomia genital.

Segundo Arrais ELM, et al. (2017), o indivíduo idoso está mais suscetível a adquirir infecção hospitalar devido às alterações do envelhecimento, declínio da resposta imunológica e realização de procedimentos invasivos, sendo que aproximadamente $80 \%$ dessas infecções estão relacionadas ao Cateterismo urinário e fatores relacionados à manutenção, manipulação, técnica de inserção, fixação e tempo de uso do dispositivo. Nesta faixa etária, pode ser esperada taxa mais alta de incidência de ITU, tanto para mulheres, quanto para os homens (BRASIL, 2003). 
Porém, a literatura mostra também que a ocorrência de ITU aumenta em homens acima de 50 anos de idade, possivelmente devido a doenças que afetam o trato geniturinário como a hipertrofia prostática, além disso, a incontinência urinária, comum nos idosos, predispõe a ITU, inclusive pelo fato de alguns necessitarem realizar o cateterismo vesical intermitente (ARRAIS ELM, et al., 2017; MELO LS, et al.,2017).

No presente estudo, o tempo médio de internação na instituição hospitalar foi de 18 dias e todas as ITU foram de origem hospitalar. Essa problemática é potencializada quando, dentre outras características, há um tempo de uso prolongado de dispositivos invasivos como o CVD (KERNKAMP CL, et al.,2016). Além disso, a técnica empregada na inserção do cateter, bem como a manutenção e esvaziamento do sistema de drenagem podem ser fatores predisponentes de infecção (BRASIL, 2017).

No que se refere aos microrganismos identificados nas uroculturas. O Proteus $s p$ e Escherichia coli são bactérias gram-negativas da flora natural humana, localizados no trato digestivo. Embora sejam bactérias de baixa virulência, podem causar infecção em indivíduos com estado clínico comprometido, denominadas de bactérias oportunistas (BRASIL, 2017).

Como demonstrado no Quadro 1, foram selecionados 5 diagnósticos de enfermagem mais frequentes, desses, 2 foram identificados como diagnósticos de enfermagem de risco. Dos diagnósticos potenciais temse: risco de infecção e risco de lesão, os quais apresentaram frequência igual a $100 \%$ nos pacientes internados.

A partir destes dados, infere-se que ambos os diagnósticos de risco podem contribuir negativamente no processo saúde-doença destes pacientes, sendo, portanto, um diferencial no cuidado a eles a implementação de medidas para que estes diagnósticos não se tornem reais. Dentre os diagnósticos reais têm-se a proteção ineficaz, que conforme o Quadro 1, o qual aparece na frequência de $100 \%$, a dor aguda com $90 \%$ de frequência e por fim, hipertermia em $90 \%$ dos casos. Entre os diagnósticos reais encontrados, observa-se que a assistência de Enfermagem precisa estar articulada com os demais profissionais de saúde para o tratamento destes sinais e sintomas na restauração da saúde do paciente.

A atuação da enfermeira tem sido crucial na prevenção e controle da infecção hospitalar relacionada à assistência em saúde, visto que é função desta assegurar que toda a equipe que realiza o cuidado ao paciente seja consciente e siga as normas no sentido de minimizar os riscos aos pacientes internados (FERREIRA AM, et al., 2016; TONINI TFF, 2013).

O diagnóstico Proteção Ineficaz é definido pela NANDA-I (2017) como diminuição na capacidade de proteger-se de ameaças internas ou externas, como doenças ou lesões. As características que definem esse diagnóstico são o envelhecimento que é considerado um processo progressivo e dinâmico, no qual modificações morfológicas, funcionais e bioquímicas interferem na capacidade funcional do indivíduo, tornando-o mais vulnerável a agravos de saúde e a doenças (HERDMAN TH, KAMITSURU S, 2017).

A população idosa pela própria característica de senescência e predisposição e maiores fatores de risco a senilidade acabam adentrando aos serviços de saúde já com alguma doença crônica instalada (ARRAIS ELM, et al., 2017). É importante destacar, que cerca de $90 \%$ dos pacientes adentram o serviço para tratar uma doença e dentro do hospital acabam contraindo uma ITU principalmente pelo uso do cateter vesical, o que se denomina Infecção Relacionada à Assistência à Saúde (BRASIL, 2017).

O conceito de Risco de Infecção é definido como "risco de ser invadido por microrganismos patogênicos". O risco de infecção está relacionado à imunodeficiência dos pacientes idosos, e a presença do CVD no trato urinário. Adicionalmente, embora já se tenha uma infecção instalada, ou não, existe a possibilidade dessa infecção se instalar ou ascender para órgãos superiores e causar uma infecção generalizada (ARRAIS ELM, et al., 2017).

As infecções representam uma ameaça significativa de morbidade e mortalidade para as pessoas idosas, em parte por causa da resposta comprometida das defesas do hospedeiro provocada por uma redução na imunidade celular e humoral. Além da perda da reserva fisiológica relacionada com a idade e as doenças crônicas também contribuem para a suscetibilidade aumentada (SMELTZER SC, BARE BG, 2012). 
O diagnóstico risco de Lesão é conceituado como "resultado de condições ambientais interagindo com os recursos adaptativos e defensivos do indivíduo" o fator relacionado é o ao trauma tissular que o paciente é exposto durante a passagem da sonda vesical de demora e consequente introdução de microrganismos no trato urinário, a não fixação correta da sonda, manejo incorreto da sonda vesical de demora são fatores de risco relevantes (HERDMAN TH, KAMITSURU S, 2017).

O diagnóstico de Hipertermia tem a seguinte definição "temperatura corporal elevada acima dos parâmetros normais". Os pacientes idosos frequentemente carecem dos sintomas típicos da ITU e sepse (LONGO DL, et al., 2013). Para tanto, é imprescindível a identificação precoce de um processo infeccioso, principalmente na presença deste sintoma, e o uso do CVD associado ao surgimento da febre pode antecipar as ações da equipe de enfermagem no reconhecimento de uma infecção e na prevenção de complicações mais graves como a sepse (TOLENTINO ACMS, 2013).

A Dor Aguda é definida como experiência sensorial e emocional desagradável que surge de lesão tissular real ou potencial ou descrita em termos de tal lesão (Associação Internacional para o Estudo da Dor); início súbito ou lento, de intensidade leve a intensa, com término antecipado ou previsível e duração de menos de seis meses (HERDMAN TH, KAMITSURU S, 2017). A dor tem sido considerada como $05^{\circ}$ sinal vital, pois isto vem assegurar que ele seja identificado, mensurado e tratado, contribuindo assim na recuperação adequada do paciente (ARAÚJO LC, ROMERO B, 2015).

A literatura aponta que o envelhecimento parece influenciar os aspectos funcionais do sistema nervoso, conforme evidenciado por uma perda de fibras mielinizadas e desmielinizadas no sistema nervoso periférico. A diminuição nas fibras mielinizadas é responsável, em parte, por provocar uma diminuição na expressão das principais proteínas da mielina. Isso causa uma redução gradual no fluxo sanguíneo endoneural com o avançar da idade, o que pode contribuir para função nervosa periférica reduzida e para a percepção diminuída da dor (LONGO DL, et al., 2013).

A NOC compreende os Resultados que descrevem o estado, comportamentos, reações e sentimentos do paciente, em resposta ao cuidado prestado. O uso da NOC possibilita, dessa maneira, monitorar a melhora, a piora ou a estagnação do estado do paciente durante um período de cuidado (MOORHEAD S, et al., 2016).

As ligações entre os resultados NOC e as intervenções NIC sugerem uma relação para a resolução do problema e as ações de enfermagem dirigidas a sua solução. A respeito das atividades e metas de enfermagem foram sugeridos intervenções e resultados que são consideradas prioritárias na prática clínica e para obtenção de uma atenção a saúde qualificada direcionada ao paciente idoso com infecção urinária (BULECHEK GM, et al.,2016; MOORHEAD S, et al., 2016).

Destaca-se o resultado controle de infecção e salienta-se que cerca de $1 / 3$ ou $1 / 2$ de todas as infecções hospitalares são preveníeis. Medidas simples como a higienização das mãos e precauções padrão são eficazes e obrigatórias no controle das Infecções Relacionadas à Assistência (BRASIL, 2017).

Conforme Ferreira AM, et al. (2016) a utilização do processo de enfermagem em todas as etapas pode fornecer entre outras vantagens, uma linguagem comum para o exercício profissional, sistematizando ações, minimizando erros, evitando perdas de tempo (FERREIRA AM, et al., 2016). Para prescrever as intervenções de enfermagem é necessário haver um diagnostico prévio e um plano de ação sobre o que deve ser prescrito, a fim de que os resultados esperados sejam alcançados. Para tanto, foi criada a Classificação Internacional das intervenções de Enfermagem (NIC), que padroniza a linguagem usada pelos enfermeiros na prescrição dos cuidados para os pacientes. As prescrições de enfermagem têm o intuito de minimizar ou solucionar os problemas apresentados pelos pacientes (BULECHEK GM, et al., 2016).

Com objetivo de reduzir os altos índices de ITU na instituição estudada, foram elaborados normas, rotinas e procedimentos sobre medidas preventivas para a ITU associada ao cateter vesical de demora, servindo de suporte aos profissionais dos serviços de saúde. 


\section{CONSIDERAÇÕES FINAIS}

O conhecimento sobre as classificações de Enfermagem na população idosa, com ITU associado ao uso do CVD é fundamental para subsidiar a assistência, bem como fundamentar as ações em bases teóricas científicas seguras. $O$ uso delas tem contribuído de forma efetiva para a valorização do conhecimento científico na Enfermagem, sustentabilidade do aporte teórico-prático e autonomia da profissão na prática assistencial tão pouco exercitada. No entanto, a produção de artigos que retratem as classificações NANDA, NOC e NIC é mínima, o que torna ainda mais desafiador difundir o conhecimento sobre elas e aplicá-las na prática assistencial, seja na atenção primária ou secundária à saúde.

\section{REFERÊNCIAS}

1. ARAÚJO LC, ROMERO B. Dor: avaliação do $5^{\circ}$ sinal vital. Uma reflexão teórica. Rev Dor, 2015; 16(4): 291- 6 .

2. ARRAIS ELM, et al. Prevenção de infecção urinária: indicadores de qualidade da assistência de enfermagem em idosos. Rev Enferm UFPE on line, 2017; 11(8): 3151-7.

3. BRASIL. Agência Nacional de Vigilância Sanitária. Segurança do paciente e Qualidade em Serviços de Saúde. Medidas de Prevenção de Infecção Relacionada à Assistência à Saúde. Brasília: Anvisa, 2017.

4. BRASIL. Lei no $10741,1^{\circ}$ de outubro de 2003. Dispõe sobre o Estatuto do Idoso e dá outras providências. Brasília, DF, out 2003. Disponível em <http://www.planalto.gov.br/ccivil_03/Leis/2003/110.741.htm>. Acesso em 20 de abril de 2019.

5. BULECHEK GM, et al. Classificação das intervenções de enfermagem - NIC. Rio de Janeiro: Elsevier, 2016; 640p.

6. COFEN (Conselho Federal de Enfermagem). Resolução COFEN n. 358, de 15 de outubro de 2009. Dispõe sobre a sistematização da assistência de Enfermagem e a implementação do Processo de Enfermagem em ambientes públicos ou privados em que ocorre o cuidado profissional de enfermagem e dá outras providências. Diário Oficial da União, Brasília (DF); 2009 out 15. Disponível em < http://www.cofen.gov.br/resoluo-cofen-3582009_4384.html>. Acesso em 20 de abril de 2019.

7. FERREIRA AM, et al. Diagnóstico de enfermagem em terapia intensiva: mapeamento cruzado e Taxonomia da NANDA-I. Rev Bras Enfermagem, 2016; 69(2): 307-315.

8. HERDMAN TH, KAMITSURU S. Diagnóstico de Enfermagem NANDA I: Definições e classificações 2018-2020. Porto Alegre: Artmed, 2017; 488p.

9. KERNKAMP CL, et al. Perfil de morbidade e gastos hospitalares com idosos no Paraná, Brasil, entre 2008 e 2012. Cad. Saúde Pública, 2016; 32(7):1-14.

10. LONGO DL, et al. Medicina interna de Harrison. São Paulo: Artmed, 2013; 4016p.

11. MELO LS, et al. Infecção do trato urinário: uma coorte de idosos com incontinência urinária. Rev Bras Enferm, 2017; 70(4): 838-844.

12. MOORHEAD S, et al. Classificação dos resultados de enfermagem - NOC. Rio de Janeiro: Elsevier, 2016; 712p.

13. SCHMITZ EL, et al. Filosofia e marco conceitual: estruturando coletivamente a sistematização da assistência de enfermagem. Rev Gaúcha Enferm, 2016; 37(esp):1-9.

14. SMELTZER SC, BARE BG. Tratado de Enfermagem Médico-Cirúrgica. Rio de Janeiro: Guanabara Koogan, 2012; 2308p.

15. TOLENTINO ACMS. O custo do cateterismo vesical de demora nos pacientes internados na unidade de terapia intensiva nos hospitais universitários. Dissertação (Mestrado em Enfermagem) - Programa de Pós-Graduação em Enfermagem. Universidade Federal do Estado do Rio de Janeiro, Rio de Janeiro, 2013; 105p.

16. TONINI TFF. O controle de infecção hospitalar em unidade de terapia intensiva: percepção de enfermeiros. Dissertação (Mestrado em Enfermagem) - Departamento de Centro de Ciências da Saúde. Universidade Federal de Santa Maria, Santa Maria, 2013; 65p. 